\title{
Broad-Band Second-Harmonic Generation in Holey Optical Fibers
}

\author{
Tanya M. Monro, V. Pruneri, N. G. R. Broderick, D. Faccio, P. G. Kazansky, and D. J. Richardson
}

\begin{abstract}
Holey fibers are shown to have an ideal geometry for efficient parametric processes due to their tailorable modal properties. These fibers can have the additional advantage of single-mode operation at all the interacting wavelengths. We demonstrate theoretically that by appropriate choice of holey fiber geometry, these fibers can be up to four orders of magnitude more efficient for second harmonic generation than conventional poled fibers.
\end{abstract}

Index Terms-Frequency conversion, nonlinear optics, optical fiber applications, optical fibers.

$\mathbf{H}$ OLEY or microstructured optical fibers (HFs) possess wavelength-scale air holes in the cladding, which lead to a unique range of optical properties. For example, these fibers can be endlessly single mode [1] and the mode area can be tailored over three orders of magnitude [2]. A range of useful dispersion regimes have also been identified such as broad-band flattened dispersion [2] and zero dispersion wavelengths shifted as low as $550 \mathrm{~nm}$ [3]. It has been demonstrated that HFs can achieve large bandwidth and small effective areas for four-wave mixing [4].

Here, we consider parametric processes involving significantly different wavelengths. We consider specifically the second-harmonic-generation (SHG) process where a fiber is pumped at $1.55 \mu \mathrm{m}$ to produce $0.775 \mu \mathrm{m}$ light. Note that SHG and parametric downconversion (PDC) can be considered as inverse processes. We show here theoretically that HFs offer significantly more freedom for producing optimum mode properties at second harmonic and pump wavelengths than conventional fibers. Previous theoretical work shows that high-NA conventional fibers can offer significant improvements in second harmonic efficiency [6]. We show here that the flexibility provided by the microstructured geometry allows up to four orders of magnitude improvement in the SHG efficiency relative to conventional poled optical fibers.

Efficient SHG requires the relative phase mismatch between fundamental and second harmonic fields to be zero, and fiber dispersion generally prevents cumulative growth of the interacting fields over the entire interaction length. One practical means for achieving phase matching is quasi-phase matching (QPM) [5]. QPM requires modulation of the fiber properties, which is typically achieved by periodically poling [7]. The

Manuscript received March 28, 2001.

The authors are with the Optoelectronics Research Centre, University of Southampton, Southampton SO17 1BJ, U.K. (e-mail: tmm@orc.soton.ac.uk). Publisher Item Identifier S 1041-1135(01)07530-9. phase matching condition for QPM SHG can be expressed in terms of modal propagation constants as $\Delta \beta=0$, where

$$
\begin{aligned}
\Delta \beta= & \beta(2 \omega)-2 \beta(\omega)-m(2 \pi / \mathcal{P}) \\
= & {\left[\beta\left(2 \omega_{0}\right)-2 \beta\left(\omega_{0}\right)-m(2 \pi / \mathcal{P})\right] } \\
& +2\left(\beta^{\prime}\left(2 \omega_{0}\right)-\beta^{\prime}\left(\omega_{0}\right)\right)\left[\omega-\omega_{0}\right] \\
& +\left(2 \beta^{\prime \prime}\left(2 \omega_{0}\right)-\beta^{\prime \prime}\left(\omega_{0}\right)\right)\left[\omega-\omega_{0}\right]^{2}+O\left[\omega-\omega_{0}\right]^{3} .
\end{aligned}
$$

The last term in (1) is the contribution of the $m$ th order harmonic when the poling period is $\mathcal{P}$. Expansion (2) shows that while QPM allows phase matching to lowest order, dispersive effects at higher orders contribute to phase mismatch between fundamental and second harmonic waves.

It can be shown that the efficiency of SHG $(\eta)$ is

$$
\eta \propto \frac{L^{2}}{A_{\text {ovl }}} \sin c^{2}\left(\frac{\Delta \beta L}{2}\right)
$$

when the nonlinearity is assumed to be uniform in the transverse plane, $L$ is the interaction length, and $A_{\text {ovl }}$ is the effective area which takes account of the overlap between interacting waves

$$
A_{\mathrm{ovl}}=\left|\iint E_{\mathrm{SH}}^{*} E_{F}^{2} d x d y\right|^{-2}
$$

where $E_{\mathrm{SH}}, E_{F}$ are normalized second harmonic and fundamental transverse modes. Although (3) is only strictly true for low-conversion efficiency processes and, for QPM, uniformly periodic structures, it gives a qualitative description. The efficiency is maximized for wavelengths that satisfy the QPM condition. We can define a bandwidth (BW) for the process as the wavelength range over which $\sin c^{2}(\Delta \beta L / 2) \geq 1 / 2$. Clearly, a longer $L$ improves $\eta$, while narrowing the usable $\mathrm{BW}$. In practice, it is desirable to maximize the $\mathrm{BW}$ without compromising efficiency. Large BW is important to ensure stable device operation or for pulsed devices for which interactions must occur for all the spectral components of the pulse.

Efficient QPM-SHG and PDC have been demonstrated in periodically poled ferroelectric waveguides [8] and glass fibers [7]. Although high efficiency was achieved, the practical application of SHG is limited by the BW and the lack of single-mode operation at all wavelengths. For QPM-PDC (and difference frequency generation) complex waveguide structures [9] are required to efficiently launch the short wavelength pump into a waveguide which is single mode for the idler and the signal. Holey fibers potentially overcome this problem by being single mode at all interacting wavelengths.

We have predicted the SH efficiency for a range of holey fibers using the model in [2], and a selection of results are 
TABLE I

Properties AND Figure of Merit FOR SHG in a RANGe of Holey Fibers $(L=10 \mathrm{~cm}$ DEVICE)

\begin{tabular}{c|ccc|ccc|c|c|c}
\hline & $\begin{array}{c}\Lambda \\
{[\mu \mathrm{m}]}\end{array}$ & $d_{\text {in }} / \Lambda$ & $d_{\text {out }} / \Lambda$ & $\begin{array}{c}\text { Overlap } \\
\text { Area }\end{array}$ & $\begin{array}{c}A_{\text {ovl }} \\
\text { Factor }\end{array}$ & $\begin{array}{c}\mathrm{BW} \\
\text { Factor }\end{array}\left[\mu \mathrm{m}^{2}\right]$ & $\begin{array}{c}\mathcal{P} \\
{[\mathrm{nm}]}\end{array}$ & $\begin{array}{c}\text { FOM } \\
{\left[\times 10^{-6}\right]}\end{array}$ \\
\hline \hline $\mathbf{A}$ & 1.0 & 0.1 & 0.1 & 0.98 & 19.1 & 18.7 & 5.8 & 44 & 1.8 \\
\hline $\mathbf{B}$ & 1.0 & 0.1 & 0.2 & 0.91 & 15.8 & 14.4 & 6.5 & 40 & 3.0 \\
\hline $\mathbf{C}$ & 1.0 & 0.15 & 0.1 & 1.02 & 20.0 & 20.4 & 15 & 43 & 11 \\
\hline $\mathbf{D}$ & 1.0 & 0.2 & 0.1 & 1.07 & 21.3 & 22.8 & 25 & 41 & 27 \\
\hline $\mathbf{E}$ & 1.0 & 0.2 & 0.4 & 0.88 & 9.4 & 8.3 & 1.6 & 27 & 0.3 \\
\hline $\mathbf{F}$ & 1.0 & 0.5 & 0.5 & 0.82 & 7.5 & 6.2 & 0.3 & 15 & 0.02 \\
\hline $\mathbf{G}$ & 2.0 & 0.4 & 0.4 & 0.87 & 12.3 & 10.7 & 89 & 30 & 720 \\
\hline $\mathbf{H}$ & 2.5 & 0.4 & 0.4 & 0.91 & 14.8 & 13.5 & 12 & 36 & 10 \\
\hline $\mathbf{I}$ & 3.0 & 0.1 & 0.1 & 0.89 & 140 & 125 & 1.8 & 67 & 0.03 \\
\hline $\mathbf{J}$ & 3.0 & 0.4 & 0.4 & 0.93 & 18.7 & 17.4 & 45 & 41 & 120 \\
\hline $\mathbf{K}$ & 3.5 & 0.4 & 0.4 & 0.94 & 23.5 & 22.1 & 53 & 46 & 128 \\
\hline $\mathbf{L}$ & 3.75 & 0.4 & 0.4 & 0.95 & 26.2 & 24.8 & 56 & 49 & 126 \\
\hline $\mathbf{M}$ & 4.0 & 0.4 & 0.4 & 0.95 & 29.0 & 27.7 & 9.5 & 51 & 3.2 \\
\hline
\end{tabular}

TABLE II

Properties and Figure of Merit For SHG in Some Conventional Fibers ( $L=10 \mathrm{~cm}$ DEVICE, $a$ IS THE CORE RADIUS)

\begin{tabular}{c|cc|cc|c|c|c}
\hline & NA & $\begin{array}{c}a \\
{[\mu \mathrm{m}]}\end{array}$ & $\begin{array}{c}A_{e f f}[\mathrm{SH}] \\
{\left[\mu m^{2}\right]}\end{array}$ & $\begin{array}{c}A_{\text {eff }}[\mathrm{F}] \\
{\left[\mu m^{2}\right]}\end{array}$ & $\begin{array}{c}A_{\text {ovt }} \\
{\left[\mu m^{2}\right]}\end{array}$ & $\begin{array}{c}\text { BW } \\
{[\mathrm{nm}]}\end{array}$ & $\begin{array}{c}\text { FOM } \\
{\left[\times 10^{-6}\right]}\end{array}$ \\
\hline \hline $\mathbf{X}$ & 0.065 & 7 & 125 & 290 & 220 & 1.8 & 0.02 \\
\hline $\mathbf{Y}$ & 0.19 & 3 & 18 & 36 & 35 & 2.0 & 0.1 \\
\hline $\mathbf{Z}$ & 0.32 & 1.8 & 6.5 & 13 & 13 & 2.2 & 0.4 \\
\hline
\end{tabular}

given in Table I. Fibers with hexagonally arranged holes of two different sizes were explored: The innermost ring of holes have diameter $d_{\text {in }}, d_{\text {out }}$ is the diameter of all other holes and $\Lambda$ is the center-to-center hole spacing. Some values for conventional fibers are included in Table II for reference. Since we require accurate values of the propagation constants at disparate wavelengths, we incorporate the wavelength-dependent refractive index of silica from the start. The structures in Table I have effective core diameters $(2 \Lambda)$ ranging from $2 \rightarrow 8 \mu \mathrm{m}$. These small core diameters are chosen because the dispersive properties can be varied more via the hole arrangement when the structure scale is comparable to the wavelength of light guided in the fiber. Also, small cores enable us to reduce $A_{\text {ovl }}$ and hence improve the SHG efficiency. When the holes are small, HFs can be endlessly single-mode, and here we consider structures which are either single or few moded. Structures of these dimensions can be readily fabricated.

Equation (2) shows that when QPM is used to eliminate the lowest order phase mismatch between fundamental and second harmonic waves, the remaining mismatch depends on the relative group velocity, group velocity dispersion, dispersion slope, etc., at each wavelength. The dispersive properties of the fibers shown in Table I are critically dependent on the cladding geometry. For example, although the material dispersion dominates at $0.775 \mu \mathrm{m}$, resulting in net normal dispersion, both normal and
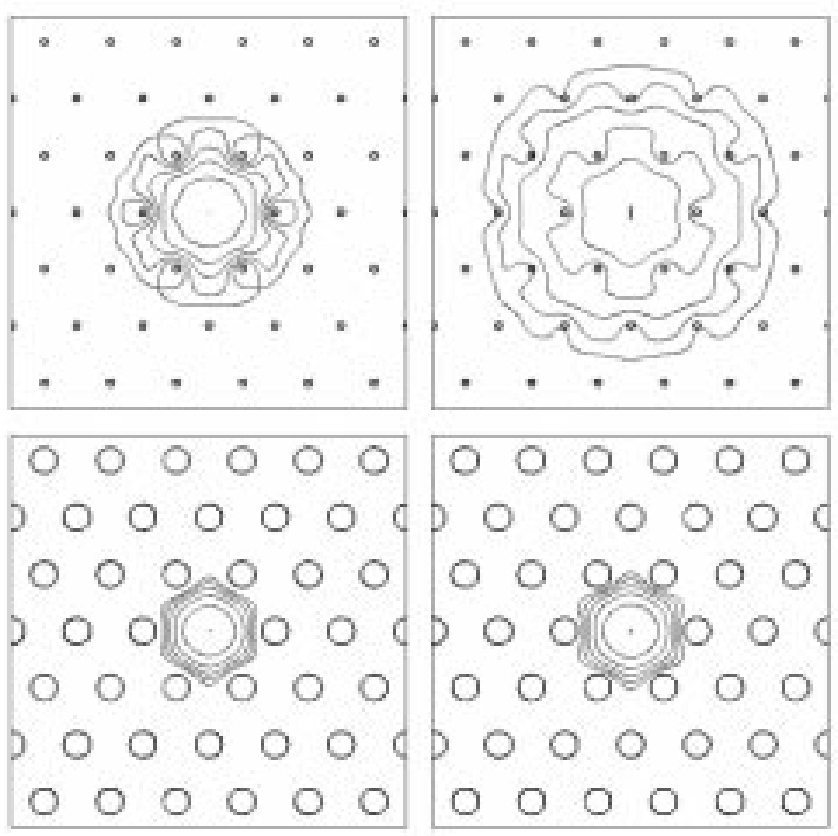

Fig. 1. Mode at $0.775 \mu \mathrm{m}$ (left), mode at $1.55 \mu \mathrm{m}$ (right) for Fiber "I" (top), Fiber "J" (bottom). Contours are separated by $2 \mathrm{~dB}$.

anomalous dispersion are possible at $1.55 \mu \mathrm{m}$. The $\mathrm{BW}$ is similarly sensitive to the fiber parameters, and indeed Table I shows that the BW can be tailored by a factor of 60 . We find that the SH BW in holey fibers can be at least 30 times broader than in comparable conventional fibers (Table II).

Another way of improving the SH efficiency $\eta$ is to reduce $A_{\text {ovl }}$, which is an effective mode area that also accounts for the overlap between fundamental and second harmonic modes. Not surprisingly, $A_{\mathrm{ovl}}$ is less sensitive to the geometry than the BW is. The value of $A_{\mathrm{ovl}}$ can be reduced by reducing the core size, and this can be seen from Table I. In addition, $A_{\text {ovl }}$ can be reduced by optimizing the overlap between fundamental and second harmonic modes. Note with reference to (4) that ideally the second harmonic field should have the same mode shape/size as the squared fundamental field in order to reduce $A_{\mathrm{ovl}}$. Both effects can be seen in Fig. 1, which shows the fundamental and second harmonic modes of fibers $\mathbf{I}$ and $\mathbf{J}$ from Table I superimposed on the fiber profiles. The larger holes in $\mathbf{J}$ confine the modes more tightly to the core, resulting in smaller modes and improved modal overlap. The contribution of the mode area and overlap factors to $A_{\text {ovl }}$ has been separated in Table I, and we conclude that for the fibers considered here, the most significant contribution to efficiency results from the reduced mode size.

Although $A_{\text {ovl }}$ can be optimized using large air holes, this does not necessarily lead to the best overall choice of structure. For example, although tight mode confinement in Fiber F leads to small $A_{\text {ovl }}$, the BW is very small. In order to obtain a measure of the suitability of any given structure for this SHG process, we construct a figure of merit (FOM) for the efficiency, $\mathrm{FOM}=\mathrm{BW}^{2} / A_{\mathrm{ovl}}$. We make this choice because for a fixed device BW, $\eta$ scales as $L^{2} / A_{\text {ovl }}$ [see (3)], and the values in Table I are calculated for a fixed length $(L=10 \mathrm{~cm})$. The 
last column in Tables I and II gives FOM for each fiber. Note that a range of holey fibers have a substantially larger FOM for SHG than the conventional fibers, and that the HF geometry can provide up to four orders of magnitude improvement to the FOM. Holey fibers such as $\mathbf{G}, \mathbf{J}, \mathbf{K}, \mathbf{L}$, which have a broad BW have a small $A_{\text {ovl }}$ relative to typical conventional fibers, and each factor contributes significantly to the overall improvement in the FOM. In the selection of fibers presented here, the best results are obtained for relatively large values of $d / \Lambda$. Note that if $\Lambda$ is small it is still possible to obtain broad-band single-mode guidance in a large air-fraction $\mathrm{HF}$.

In [7], 20\% efficiency was demonstrated for SHG at $1532 \mathrm{~nm}$ in Fiber Y (Table II) using $L=7.5 \mathrm{~cm}$ and $4 \mathrm{~kW}$ peak fundamental power. This represents the best result to date in conventional fibers. When the BW is not critical, a small $A_{\text {ovl }}$ significantly reduces the power requirements for SHG. For example, Fiber $\mathbf{F}$ has $A_{\text {ovl }}=6.2 \mu \mathrm{m}^{2}$. Taking into account the different device lengths in each case ( $L=7.5 \mathrm{~cm}$ for $\mathbf{Y}, 10 \mathrm{~cm}$ for $\mathbf{F}$ ), approximately ten times less power is required to achieve the same efficiency in Fiber $\mathbf{F}$ than in Fiber $\mathbf{Y}$, reducing the power levels to $\approx 100 \mathrm{~W}$. Similarly, for BW critical applications, Fiber $\mathbf{G}$ requires approximately six times less fundamental power to achieve the same efficiency as Fiber $\mathbf{Y}$ while increasing the assessable phase-matching BW from 2 to $90 \mathrm{~nm}$, which allows for flexible device design. These examples demonstrate the advantages holey fibers can offer over conventional technology.

Recently, we have demonstrated experimentally that holey fibers can be poled [10]. This demonstrates that the complex air : glass geometry in a holey fiber is compatible with thermal poling techniques. Note that metal electrodes are typically required for poling, and since holey fibers are generally fabricated by stacking tubes, it is straightforward to integrate these electrodes into the structure, which should allow for efficient poling. Table I shows that these HFs require QPM periods $(\mathcal{P})$ ranging from 28 to $67 \mu \mathrm{m}$, large enough to be easily produced.

In conclusion, we predict that by using a holey or microstructured optical fiber, the efficiency of phase-matching can be improved for second-harmonic generation. As well as offering endless single-mode guidance, the dispersion, mode size and modal overlap can be optimized to improve the efficiency and usefulness of the SHG process. Combining these factors, we demonstrate that holey fibers can offer up to four orders of magnitude improvements for SHG over conventional fiber designs. Future investigations into other air hole arrangements are likely to yield further improvements to SHG efficiency, and similar improvements should also be possible for parametric processes other than SHG and PDC.

\section{REFERENCES}

[1] T. A. Birks, J. C. Knight, and P. St. J. Russell, "Endlessly single-mode photonic crystal fiber," Opt. Lett., vol. 22, pp. 961-963, 1997.

[2] T. M. Monro, D. J. Richardson, N. G. R. Broderick, and P. J. Bennett, "Holey optical fibers: An efficient modal model," J. Lightwave Technol., vol. 17, pp. 1093-1102, June 1999.

[3] J. C. Knight, J. Arriaga, T. A. Birks, A. Ortigosa-Blanch, W. J. Wadsworth, and P. St. J. Russell, "Anomalous dispersion in photonic crystal fiber," IEEE Photon. Technol. Lett., vol. 12, pp. 807-809, July 2000.

[4] J. K. Ranka, R. S. Windeler, and A. J. Stentz, "Optical properties of high-delta air-silica microstructure optical fibers," Opt. Lett., vol. 25, no. 11 , pp. 796-798, 2000.

[5] J. A. Armstrong, N. Bloembergen, J. Ducuing, and P. S. Pershan, "Interactions between light waves in a nonlinear dielectric," Phys. Rev., vol. 127, pp. 1918-1939, 1962

[6] A. Arraf and C. M. de Sterke, "Large bandwidth frequency conversion in high-NA step-index optical fibers," J. Quantum Electron., vol. 34, pp. 660-665, Apr. 1998.

[7] V. Pruneri, G. Bonfrate, P. G. Kazansky, D. J. Richardson, N. G. Broderick, J. P. deSandro, C. Simonneau, P. Vidakovic, and J. A. Levenson, "Greater than 20 quasiphase-matched germanosilicate optical fibers," Opt. Lett., vol. 24, pp. 208-210, 1999.

[8] M. Yamada, N. Nada, M. Saitoh, and K. Watanabe, "1st-order quasiphase matched $\mathrm{LiNbO}_{3}$ wave-guide periodically poled by applying an external-field for efficient blue 2nd-harmonic generation," Appl. Phys. Lett., vol. 62, pp. 435-436, 1993.

[9] M. H. Chou, J. Hauden, M. A. Arbore, and M. M. Fejer, " $1.5-\mu \mathrm{m}-b a n d$ wavelength conversion based on difference-frequency generation in $\mathrm{LiNbO}_{3}$ waveguides with integrated coupling structures," Opt. Lett., vol. 23, pp. 1004-1006, 1998.

[10] D. Faccio, A. Busacca, W. Belardi, V. Pruneri, P. G. Kazansky, T. M. Monro, D. J. Richardson, B. Grappe, M. Cooper, and C. N. Pannell, "Demonstration of thermal poling in holey fibers," Electron. Lett., vol. 37, no. 2, pp. 107-108, 2001. 